\title{
Synthesis of Self-Aggregative Zinc Chlorophylls Possessing Polymerizable Esters as a Stable Model Compound for Main Light-Harvesting Antennas of Green Photosynthetic Bacteria
}

\author{
Hitoshi Tamiaki, Kazuya Nishihara, and Reiko Shibata \\ Department of Bioscience and Biotechnology, Faculty of Science and Engineering, Ritsumeikan University, \\ Shiga 525 8577, Kusatsu, Japan
}

Received 24 June 2006; Revised 19 July 2006; Accepted 28 August 2006

\begin{abstract}
Zinc bacteriochlorophyll- $d$ derivatives possessing a polymerizable moiety at the 17-propionate were prepared as model compounds of natural occurring chlorophylls in the main peripheral antennas of green photosynthetic bacteria (chlorosomes). The synthetic compounds self-aggregated in nonpolar organic solvents as well as in the solid state to give large oligomers similar to chlorosomal J-aggregates. Such introduction of the polymerizable groups in the ester did not suppress the ability of selfaggregation.
\end{abstract}

Copyright (C) 2006 Hitoshi Tamiaki et al. This is an open access article distributed under the Creative Commons Attribution License, which permits unrestricted use, distribution, and reproduction in any medium, provided the original work is properly cited.

\section{INTRODUCTION}

Green photosynthetic bacteria have extramembranous peripheral antenna systems, called chlorosomes. Chlorosomes are main light-harvesting antennas in the cell and are different from any other antennas in the structural motif. Chlorosomal core parts are built by self-aggregation of composite chlorophyllous pigments [1-5], while the others are based on specific interaction of (bacterio)chlorophylls with peptides. In chlorosomes of Chloroflexus aurantiacus, one of the green nonsulfur (filamentous or gliding/sliding) bacteria, bacteriochlorophyll(BChl)s-c possessing ethyl and methyl groups at the 8- and 12-positions, respectively, (see Figure 1(a)) are the light-absorbing chlorophyllous pigments. A large number of the molecules self-aggregate to form oligomers in a hydrophobic environment surrounded by a chlorosomal lipid monolayer. The J-aggregates with their unique ability of fast light energy migration are assumed to make rod structures in a chlorosome. In the rod model, the propionate ester group at the 17-position is situated at the surface. This location was first proposed from molecular calculation (MM+/PM3) [6] and is supported using synthetic models (Figure 1(b)) [2, 7-10]. Recently, atomic force microscopic observation showed that self-aggregates of the model compound indeed made rod structures [11].

Chlorosomal self-aggregates are fragile and dissociate readily to monomers by addition of coordinatable species.
In natural systems, the surface monolayer of lipids is reinforced by membranous additives including proteins [12] in order to suppress such deaggregation. In artificial micelle systems, amphiphilic compounds surrounding selfaggregates were cross-linked to overcome the instability of supramolecules [13-15]. Here we report synthesis of zinc bacteriochlorophyll- $d$ derivatives 3 possessing polymerizable substituents at the ester group of the 17-propionate and their self-aggregation in a nonpolar organic solvent as chlorosomal hydrophobic environments. These functional groups are assumed to be situated at the surface of the selfaggregated supramolecules and would be polymerized via $\mathrm{C}-\mathrm{C}$ bondings to stabilize the noncovalently self-assembled, large oligomeric J-aggregates [16, 17], which is promising for construction of photoactive nanodevices.

\section{EXPERIMENTAL}

\subsection{General}

All the apparatuses and solvents used were described previously [9]. Pyropheophorbide-d (6) was prepared according to the previous report [18]. 3-Acetyl-3-devinylpyropheophorbide- $a$ (9) was synthesized by similar acidic hydrolysis of the corresponding methyl ester $8[19,20](94 \%$ yield) as in preparation of $\mathbf{5} \rightarrow \mathbf{6}[18,21] .10,12$-Pentacosadiyn-1-ol, tetracosanol, and 4-hydroxybutyl acrylate were 


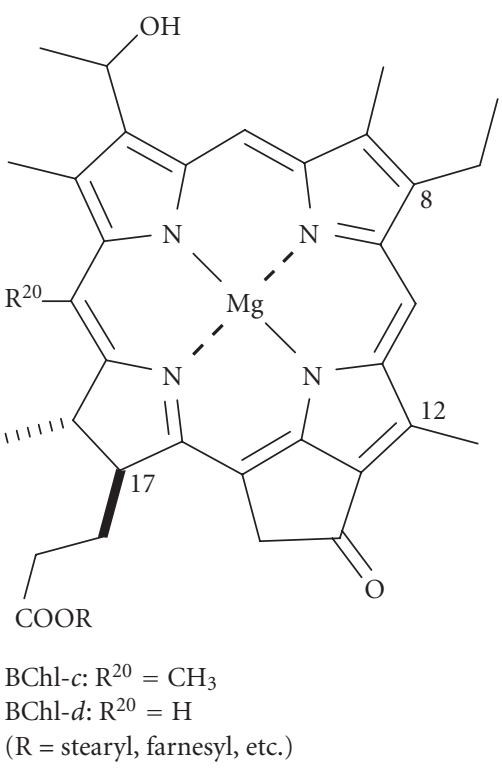

(a)

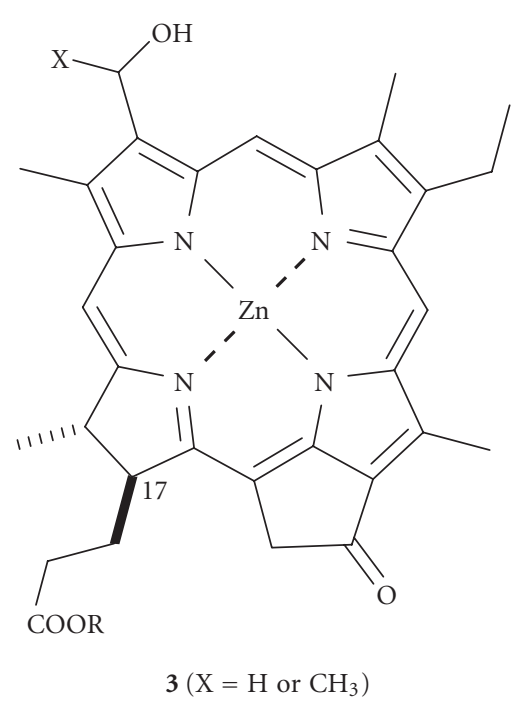

(b)

FIGURE 1: (a) Naturally occurring chlorosomal chlorophylls and (b) their synthetic models 3.

purchased from Tokyo Chemical Ind. All the reactions were done in the dark under nitrogen. TLC and flash column chromatography (FCC) were performed on $0.25 \mathrm{~mm}$ precoated silica gel $60 \mathrm{~F}_{254}$ (Merck) and silica gel, Kieselgel 60 (Merck, 40-63 $\mu \mathrm{m}$ ), respectively.

\subsection{Synthetic procedures}

\subsubsection{Esterification}

A primary alcohol $(0.3 \mathrm{mmol})$ was added to an ice-chilled dry $\mathrm{CH}_{2} \mathrm{Cl}_{2}$ solution $(30 \mathrm{~mL}$ ) of 3-carbonyl-172-carboxychlorin $\mathbf{6}$ or $9(0.1 \mathrm{mmol})$ with stirring. After further addition of 3-ethyl-1-[3-( $N, N$-dimethylamino)propyl] carbodiimide hydrochloride $(\mathrm{EDC} \cdot \mathrm{HCl}, 0.5 \mathrm{mmol})$ and $4-(N, N$ dimethylamino)pyridine (DMAP, $1 \mathrm{mmol}$ ), the solution was stirred at $0^{\circ} \mathrm{C}$ for 30 minutes and at room temperature overnight. The reaction was monitored by TLC and the complete disappearance of the starting carboxylic acid was confirmed. The reaction mixture was washed with aqueous $1 \%$ $\mathrm{HCl}, 4 \% \mathrm{NaHCO}_{3}$ and water, and dried over $\mathrm{Na}_{2} \mathrm{SO}_{4}$. The mixture was purified with FCC and recrystallization to afford the desired ester $\mathbf{1}$ as a dark brown solid.

\subsubsection{Reduction of 3-formyl group}

Tert-Butylamine borane complex $(0.25 \mathrm{mmol})$ was added to an ice-chilled dry $\mathrm{CH}_{2} \mathrm{Cl}_{2}$ solution $\left(30 \mathrm{~mL}\right.$ ) of 3-formyl-13 $3^{1}$ oxo-chlorin $1 \mathbf{a} / \mathbf{b} / \mathbf{d}(0.1 \mathrm{mmol})$, which was stirred at $0^{\circ} \mathrm{C}$ for 15 minutes and at room temperature for a few hours. The reaction was monitored by visible absorption spectra: a blue shift of $\mathrm{Q}_{\mathrm{y}}$-band from 695 to $661 \mathrm{~nm}$. After the complete shift (usually with 2-hour stirring), the reaction mixture was stirred with aqueous $2 \% \mathrm{HCl}$ solution $(10 \mathrm{~mL})$. The sepa- rated $\mathrm{CH}_{2} \mathrm{Cl}_{2}$ phase was washed with aqueous $4 \% \mathrm{NaHCO}_{3}$ and water, and dried over $\mathrm{Na}_{2} \mathrm{SO}_{4}$. The mixture was purified with FCC $\left(5 \% \mathrm{Et}_{2} \mathrm{O}-\mathrm{CH}_{2} \mathrm{Cl}_{2}\right)$ and recrystallization $\left(\mathrm{CH}_{2} \mathrm{Cl}_{2}\right.$-hexane) to afford the desired $3^{1}$-hydroxy-13 $13^{1}$-oxochlorin $\mathbf{2 a / b} / \mathbf{d}$ (black solid) as a selectively reduced product.

\subsubsection{Reduction of 3-acetyl group}

A methanol solution $(1 \mathrm{~mL})$ of sodium borohydride $(0.15$ mmol) was added to an ice-chilled dry $\mathrm{CH}_{2} \mathrm{Cl}_{2}$ solution $(30 \mathrm{~mL})$ of 3 -acetyl- $13^{1}$-oxo-chlorin $1 \mathrm{c}(0.1 \mathrm{mmol})$ with stirring. The reaction was monitored by visible absorption spectra: a blue shift of $\mathrm{Q}_{\mathrm{y}}$-band from 683 to $661 \mathrm{~nm}$. [Caution] Prolonged reaction induced an undesired reduction of the 13-keto carbonyl group. After the complete shift (usually with 10-minute stirring), the reaction was quenched by addition of acetone, washed with water, and dried over $\mathrm{Na}_{2} \mathrm{SO}_{4}$. The mixture was purified with FCC $\left(5 \% \mathrm{Et}_{2} \mathrm{O}-\mathrm{CH}_{2} \mathrm{Cl}_{2}\right)$ and recrystallization $\left(\mathrm{CH}_{2} \mathrm{Cl}_{2}-\mathrm{MeOH}\right)$ to afford the desired $3^{1}$ hydroxy-13 $1{ }^{1}$-oxo-chlorin 2 c (black solid) as a selectively reduced product.

\subsubsection{Zinc metallation}

A methanol solution $(4 \mathrm{~mL})$ saturated with zinc acetate dihydrate was added to a dry $\mathrm{CH}_{2} \mathrm{Cl}_{2}$ solution $(30 \mathrm{~mL})$ of metal-free chlorin $2(0.1 \mathrm{mmol})$, which was stirred at room temperature for 90 minutes. The reaction was monitored by TLC and the complete disappearance of the starting free base was confirmed. The reaction mixture was stirred with aqueous $4 \% \mathrm{NaHCO}_{3}$ solution $(10 \mathrm{~mL})$ for 20 minutes. The separated $\mathrm{CH}_{2} \mathrm{Cl}_{2}$ phase was washed with aqueous $4 \%$ 
$\mathrm{NaHCO}_{3}$ and water, and dried over $\mathrm{Na}_{2} \mathrm{SO}_{4}$. The mixture was purified with FCC $\left(10 \% \mathrm{Et}_{2} \mathrm{O}-\mathrm{CH}_{2} \mathrm{Cl}_{2}\right)$ and recrystallization $\left(\mathrm{CH}_{2} \mathrm{Cl}_{2}-\mathrm{MeOH}\right)$ to afford the desired zinc complex 3 as a dark green solid.

\subsection{Synthesis of chlorins}

\subsubsection{Synthesis of 10,12-pentacosadiyn-1-yl pyropheophorbide-d (1a)}

Esterification of $\mathbf{6}$ with 10,12-pentacosadiyn-1-ol gave 1a (61\% yield) after FCC $\left(1 \% \mathrm{Et}_{2} \mathrm{O}-\mathrm{CH}_{2} \mathrm{Cl}_{2}\right)$ and recrystallization $\left(\mathrm{CH}_{2} \mathrm{Cl}_{2}\right.$-hexane); VIS $\left(\mathrm{CH}_{2} \mathrm{Cl}_{2}\right) \lambda_{\max }=694$ (relative intensity, 0.80), 633 (0.08), 554 (0.16), 522 (0.14), 429 (1.00), $388 \mathrm{~nm}$ (0.83); IR (KBr) $v=1732\left(17^{2}-\mathrm{C}=\mathrm{O}\right), 1701$ $(13-\mathrm{C}=\mathrm{O}), 1678(3-\mathrm{C}=\mathrm{O}), 1618 \mathrm{~cm}^{-1} ;{ }^{1} \mathrm{H}-\mathrm{NMR}\left(\mathrm{CDCl}_{3}\right)$ $\delta=11.56(1 \mathrm{H}, \mathrm{s}, \mathrm{CHO}), 10.33,9.63,8.85($ each $1 \mathrm{H}, \mathrm{s}$, 5-, 10-, 20-H), 5.36, 5.20 (each $1 \mathrm{H}, \mathrm{d}, J=20 \mathrm{~Hz}, 13^{1}$ $\left.\mathrm{CH}_{2}\right), 4.58(1 \mathrm{H}, \mathrm{br}-\mathrm{q}, J=7 \mathrm{~Hz}, 18-\mathrm{H}), 4.40(1 \mathrm{H}, \mathrm{br}-\mathrm{d}$, $J=8 \mathrm{~Hz}, 17-\mathrm{H}), 3.98,3.91($ each $1 \mathrm{H}, \mathrm{dt}, J=11,7 \mathrm{~Hz}$, $\left.\mathrm{COOCH}_{2}\right), 3.74\left(2 \mathrm{H}, \mathrm{q}, J=7.5 \mathrm{~Hz}, 8-\mathrm{CH}_{2}\right), 3.79,3.73$, 3.33 (each $3 \mathrm{H}, \mathrm{s}, 2-, 7-, 12-\mathrm{CH}_{3}$ ), 2.67-2.78, 2.52-2.62, 2.24$2.39\left(1 \mathrm{H}+1 \mathrm{H}+2 \mathrm{H}, \mathrm{m}, 17-\mathrm{CH}_{2} \mathrm{CH}_{2}\right), 2.20,2.18($ each $2 \mathrm{H}, \mathrm{t}$, $\left.J=7 \mathrm{~Hz}, \mathrm{CH}_{2} \mathrm{C} \equiv \mathrm{C}-\mathrm{C} \equiv \mathrm{CCH}_{2}\right), 1.85(3 \mathrm{H}, \mathrm{d}, J=7 \mathrm{~Hz}, 18-$ $\left.\mathrm{CH}_{3}\right), 1.72\left(3 \mathrm{H}, \mathrm{t}, J=7.5 \mathrm{~Hz}, 8^{1}-\mathrm{CH}_{3}\right), 1.38-1.50(6 \mathrm{H}, \mathrm{m}$, $\left.\mathrm{COOCCH}_{2}, \mathrm{CH}_{2} \mathrm{CC} \equiv \mathrm{C}-\mathrm{C} \equiv \mathrm{CCCH}_{2}\right), 1.16-1.30(28 \mathrm{H}, \mathrm{m}$, $\left.17^{6-10,19-27}-\mathrm{CH}_{2}\right), 0.86\left(3 \mathrm{H}, \mathrm{t}, J=6.5 \mathrm{~Hz}, 17^{28}-\mathrm{CH}_{3}\right), 0.12$, -2.05 (each $1 \mathrm{H}, \mathrm{s}, \mathrm{NH}$ ). MS (FAB) found: $m / z 879$. Calculated for $\mathrm{C}_{57} \mathrm{H}_{75} \mathrm{~N}_{4} \mathrm{O}_{4}: \mathrm{MH}^{+}, 879$.

\subsubsection{Synthesis of 10,12-pentacosadiyn-1-y/ 3- hydroxymethyl-3-devinyl-pyropheophorbide-a (2a)}

Reduction of $\mathbf{1 a}$ with $\mathrm{t}-\mathrm{BuNH}_{2} \mathrm{BH}_{3}$ gave $\mathbf{2 a}$ ( $92 \%$ yield); VIS $\left(\mathrm{CH}_{2} \mathrm{Cl}_{2}\right) \lambda_{\max }=661$ (rel., 0.48), 605 (0.08), 535 (0.09), 504 (0.10), $409 \mathrm{~nm}$ (1.00); IR (KBr) $v=3350\left(3^{1}-\right.$ $\mathrm{O}-\mathrm{H}), 1732\left(17^{2}-\mathrm{C}=\mathrm{O}\right), 1692(13-\mathrm{C}=\mathrm{O}), 1622 \mathrm{~cm}^{-1} ;{ }^{1} \mathrm{H}-$ $\operatorname{NMR}\left(\mathrm{CDCl}_{3}\right) \delta=9.51,9.45,8.57$ (each 1H, s, 5-, 10-, 20$\mathrm{H}), 5.92\left(2 \mathrm{H}, \mathrm{s}, 3-\mathrm{CH}_{2}\right), 5.26,5.10($ each $1 \mathrm{H}, \mathrm{d}, J=20 \mathrm{~Hz}$, $\left.13^{1}-\mathrm{CH}_{2}\right), 4.49(1 \mathrm{H}$, br-q, $J=7 \mathrm{~Hz}, 18-\mathrm{H}), 4.30(1 \mathrm{H}$, br$\mathrm{d}, J=8 \mathrm{~Hz}, 17-\mathrm{H}), 3.99,3.93($ each $1 \mathrm{H}, \mathrm{dt}, J=11,7 \mathrm{~Hz}$, $\left.\mathrm{COOCH}_{2}\right), 3.70\left(2 \mathrm{H}, \mathrm{q}, J=7.5 \mathrm{~Hz}, 8-\mathrm{CH}_{2}\right), 3.67,3.49$, 3.43 (each $3 \mathrm{H}, \mathrm{s}, 2-$, 7-, 12- $\mathrm{CH}_{3}$ ), 2.62-2.73, 2.49-2.59, 2.18$2.36\left(1 \mathrm{H}+1 \mathrm{H}+2 \mathrm{H}, \mathrm{m}, 17-\mathrm{CH}_{2} \mathrm{CH}_{2}\right), 2.21,2.17($ each $2 \mathrm{H}, \mathrm{t}$, $\left.J=7 \mathrm{~Hz}, \mathrm{CH}_{2} \mathrm{C} \equiv \mathrm{C}-\mathrm{C} \equiv \mathrm{CCH}_{2}\right), 1.81(3 \mathrm{H}, \mathrm{d}, J=7 \mathrm{~Hz}, 18-$ $\left.\mathrm{CH}_{3}\right), 1.70\left(3 \mathrm{H}, \mathrm{t}, J=7.5 \mathrm{~Hz}, 8^{1}-\mathrm{CH}_{3}\right), 1.38-1.50(6 \mathrm{H}, \mathrm{m}$, $\left.\mathrm{COOCCH}_{2}, \mathrm{CH}_{2} \mathrm{CC} \equiv \mathrm{C}-\mathrm{C} \equiv \mathrm{CCCH}_{2}\right), 1.17-1.31(28 \mathrm{H}, \mathrm{m}$, $\left.17^{6-10,19-27}-\mathrm{CH}_{2}\right), 0.87\left(3 \mathrm{H}, \mathrm{t}, J=6.5 \mathrm{~Hz}, 17^{28}-\mathrm{CH}_{3}\right), 0.29$, -1.79 (each $1 \mathrm{H}, \mathrm{s}, \mathrm{NH}$ ). MS (FAB) found: $m / z 881$. Calculated for $\mathrm{C}_{57} \mathrm{H}_{77} \mathrm{~N}_{4} \mathrm{O}_{4}: \mathrm{MH}^{+}, 881$.

\subsubsection{Synthesis of zinc 10,12-pentacosadiyn-1-yl 3-hydrox- ymethyl-3-devinyl-pyropheophorbide-a (3a)}

Zinc-metallation of 2 a gave $3 \mathbf{a}\left(92 \%\right.$ yield); $\mathrm{mp}>300^{\circ} \mathrm{C}$; VIS (THF) $\lambda_{\max }=646$ (rel., 0.74), $602(0.10), 565$ (0.05), 520
(0.03), 424 (1.00), $404 \mathrm{~nm}(0.57)$; IR (KBr) $v=3150\left(3^{1}-\right.$ $\mathrm{O}-\mathrm{H}), 1732\left(17^{2}-\mathrm{C}=\mathrm{O}\right), 1664(13-\mathrm{C}=\mathrm{O}), 1620 \mathrm{~cm}^{-1} ;{ }^{1} \mathrm{H}-$ NMR $\left(\mathrm{CDCl}_{3}-1 \% \mathrm{C}_{5} \mathrm{D}_{5} \mathrm{~N}\right) \delta=9.57,9.31,8.36($ each $1 \mathrm{H}$, s, 5-, 10-, 20-H), $5.83\left(2 \mathrm{H}, \mathrm{s}, 3-\mathrm{CH}_{2}\right), 5.19,5.05($ each $1 \mathrm{H}$, $\left.\mathrm{d}, J=20 \mathrm{~Hz}, 13^{1}-\mathrm{CH}_{2}\right), 4.40(1 \mathrm{H}, \mathrm{br}-\mathrm{q}, J=7 \mathrm{~Hz}, 18-\mathrm{H})$, $4.20(1 \mathrm{H}$, br-d, $J=8 \mathrm{~Hz}, 17-\mathrm{H}), 3.95\left(2 \mathrm{H}, \mathrm{m}, \mathrm{COOCH}_{2}\right)$, $3.76\left(2 \mathrm{H}, \mathrm{q}, J=7.5 \mathrm{~Hz}, 8-\mathrm{CH}_{2}\right), 3.69,3.35,3.26($ each $3 \mathrm{H}$, s, 2-, 7-, 12- $\mathrm{CH}_{3}$ ), 2.48-2.62, 2.29-2.42 (each 2H, m, 17$\left.\mathrm{CH}_{2} \mathrm{CH}_{2}\right), 2.20\left(4 \mathrm{H}, \mathrm{m}, \mathrm{CH}_{2} \mathrm{C} \equiv \mathrm{C}-\mathrm{C} \equiv \mathrm{CCH}_{2}\right), 1.72(3 \mathrm{H}$, $\left.\mathrm{d}, J=7 \mathrm{~Hz}, 18-\mathrm{CH}_{3}\right), 1.70\left(3 \mathrm{H}, \mathrm{t}, J=7.5 \mathrm{~Hz}, 8^{1}-\mathrm{CH}_{3}\right)$, 1.41-1.50 (6H, m, $\left.\mathrm{COOCCH}_{2}, \mathrm{CH}_{2} \mathrm{CC} \equiv \mathrm{C}-\mathrm{C} \equiv \mathrm{CCCH}_{2}\right)$, $1.20-1.30\left(28 \mathrm{H}, \mathrm{m}, 17^{6-10,19-27}-\mathrm{CH}_{2}\right), 0.87(3 \mathrm{H}, \mathrm{t}, J=$ $6.5 \mathrm{~Hz}, 17^{28}-\mathrm{CH}_{3}$ ). MS (FAB) found: $m / z$ 942. Calculated for $\mathrm{C}_{57} \mathrm{H}_{74} \mathrm{~N}_{4} \mathrm{O}_{4}^{64} \mathrm{Zn}: \mathrm{M}^{+}, 942$.

\subsubsection{Synthesis of tetracosanyl pyropheophorbide-d (1b)}

Esterification of $\mathbf{6}$ with tetracosanol gave $\mathbf{1 b}$ (76\% yield) after FCC $\left(1 \% \quad \mathrm{Et}_{2} \mathrm{O}-\mathrm{CH}_{2} \mathrm{Cl}_{2}\right)$ and recrystallization $\left(\mathrm{CH}_{2} \mathrm{Cl}_{2}\right.$-hexane); ${ }^{1} \mathrm{H}-\mathrm{NMR}\left(\mathrm{CDCl}_{3}\right) \delta=11.57(1 \mathrm{H}$, s, 3-CHO), 10.33, 9.64, 8.85 (each 1H, s, 5-, 10-, 20-H), $5.35,5.20\left(\right.$ each $\left.1 \mathrm{H}, \mathrm{d}, J=20 \mathrm{~Hz}, 13^{1}-\mathrm{CH}_{2}\right), 4.58(1 \mathrm{H}$, br-q, $J=7 \mathrm{~Hz}, 18-\mathrm{H}), 4.40(1 \mathrm{H}, \mathrm{br}-\mathrm{d}, J=8 \mathrm{~Hz}, 17-\mathrm{H})$, 3.88-4.01 (2H, m, COOCH 2$), 3.79,3.73,3.34$ (each $3 \mathrm{H}, \mathrm{s}$, 2-, 7-, 12- $\left.\mathrm{CH}_{3}\right), 3.74\left(2 \mathrm{H}, \mathrm{q}, J=7 \mathrm{~Hz}, 8-\mathrm{CH}_{2}\right), 2.62-2.80$, 2.62-2.50, 2.20-2.40 (1H + 1H + 2H, m, 17- $\left.\mathrm{CH}_{2} \mathrm{CH}_{2}\right), 1.85$ $\left(3 \mathrm{H}, \mathrm{d}, J=7 \mathrm{~Hz}, 18-\mathrm{CH}_{3}\right), 1.73\left(3 \mathrm{H}, \mathrm{t}, J=7 \mathrm{~Hz}, 8^{1}-\mathrm{CH}_{3}\right)$, $1.44\left(2 \mathrm{H}, \mathrm{m}, \mathrm{COOCCH}_{2}\right), 1.05-1.36\left(42 \mathrm{H}, \mathrm{m}, 17^{6-26}-\mathrm{CH}_{2}\right)$, $0.87\left(3 \mathrm{H}, \mathrm{t}, \mathrm{J}=6 \mathrm{~Hz}, 17^{27}-\mathrm{CH}_{3}\right),-0.11,-2.04($ each $1 \mathrm{H}, \mathrm{s}$, $\mathrm{NH})$.

\subsubsection{Synthesis of tetracosanyl 3-hydroxymethyl-3-devinyl- pyropheophorbide-a (2b)}

Reduction of $\mathbf{2} \mathbf{a}$ with $\mathrm{t}-\mathrm{BuNH}_{2} \mathrm{BH}_{3}$ gave $\mathbf{2 b}$ (65\% yield); ${ }^{1} \mathrm{H}$ $\operatorname{NMR}\left(\mathrm{CDCl}_{3}\right) \delta=9.51,9.45,8.57($ each $1 \mathrm{H}, \mathrm{s}, 5-, 10-, 20-$ $\mathrm{H}), 5.92\left(2 \mathrm{H}, \mathrm{d}, J=5 \mathrm{~Hz}, 3-\mathrm{CH}_{2}\right), 5.25,5.10($ each $1 \mathrm{H}, \mathrm{d}$, $\left.J=20 \mathrm{~Hz}, 13^{1}-\mathrm{CH}_{2}\right), 4.73(1 \mathrm{H}$, br-q, $J=7 \mathrm{~Hz}, 18-\mathrm{H}), 4.29$ $(1 \mathrm{H}$, br-d, $J=8 \mathrm{~Hz}, 17-\mathrm{H}), 3.90-4.04\left(2 \mathrm{H}, \mathrm{m}, \mathrm{COOCH}_{2}\right)$, $3.70\left(2 \mathrm{H}, \mathrm{q}, J=7 \mathrm{~Hz}, 8-\mathrm{CH}_{2}\right), 3.66,3.43,3.27$ (each $3 \mathrm{H}, \mathrm{s}$, 2-, 7-, 12- $\left.\mathrm{CH}_{3}\right), 2.62-2.74,2.47-2.61,2.20-2.39,2.10-2.19$ (each $\left.1 \mathrm{H}, \mathrm{m}, 17-\mathrm{CH}_{2} \mathrm{CH}_{2}\right), 1.80\left(3 \mathrm{H}, \mathrm{d}, J=7 \mathrm{~Hz}, 18-\mathrm{CH}_{3}\right)$, $1.70\left(3 \mathrm{H}, \mathrm{t}, J=7 \mathrm{~Hz}, 8^{1}-\mathrm{CH}_{3}\right), 1.46\left(2 \mathrm{H}, \mathrm{m}, \mathrm{COOCCH}_{2}\right)$, $1.10-1.31\left(42 \mathrm{H}, \mathrm{m}, 17^{6-26}-\mathrm{CH}_{2}\right), 0.87\left(3 \mathrm{H}, \mathrm{t}, J=6 \mathrm{~Hz}, 17^{27}-\right.$ $\left.\mathrm{CH}_{3}\right), 0.30,-1.79$ (each $\left.1 \mathrm{H}, \mathrm{s}, \mathrm{NH}\right)$.

\subsubsection{Synthesis of zinc tetracosanyl 3-hydroxymethyl-3- devinyl-pyropheophorbide-a (3b)}

Zinc-metallation of $\mathbf{2} \mathbf{b}$ gave $\mathbf{3 b}$ (66\% yield); ${ }^{1} \mathrm{H}-\mathrm{NMR}$ $\left(\mathrm{CDCl}_{3}-1 \% \mathrm{C}_{5} \mathrm{D}_{5} \mathrm{~N}\right) \delta=9.56,9.31,8.33($ each $1 \mathrm{H}, \mathrm{s}, 5-, 10-$, 20-H), $5.82\left(2 \mathrm{H}, \mathrm{s}, 3-\mathrm{CH}_{2}\right), 5.17,5.05$ (each $1 \mathrm{H}, \mathrm{d}, J=20 \mathrm{~Hz}$, $\left.13^{1}-\mathrm{CH}_{2}\right), 4.38(1 \mathrm{H}$, br-q, $J=8 \mathrm{~Hz}, 18-\mathrm{H}), 4.19(1 \mathrm{H}, \mathrm{br}-\mathrm{d}$, $J=7 \mathrm{~Hz}, 17-\mathrm{H}), 3.96-3.91\left(2 \mathrm{H}, \mathrm{m}, \mathrm{COOCH}_{2}\right), 3.74(2 \mathrm{H}$, q, $\left.J=8 \mathrm{~Hz}, 8-\mathrm{CH}_{2}\right), 3.68,3.32,3.23($ each $3 \mathrm{H}, \mathrm{s}, 2-, 7-, 12-$ $\left.\mathrm{CH}_{3}\right), 2.43-2.61,1.89-2.43\left(1 \mathrm{H}+3 \mathrm{H}, \mathrm{m}, 17-\mathrm{CH}_{2} \mathrm{CH}_{2}\right), 1.70$ 
$\left(3 \mathrm{H}, \mathrm{d}, J=8 \mathrm{~Hz}, 18-\mathrm{CH}_{3}\right), 1.69\left(3 \mathrm{H}, \mathrm{t}, J=8 \mathrm{~Hz}, 8^{1}-\mathrm{CH}_{3}\right)$, $1.47\left(2 \mathrm{H}, \mathrm{m}, \mathrm{COOCCH}_{2}\right), 1.10-1.31\left(42 \mathrm{H}, \mathrm{m}, 17^{6-26}-\mathrm{CH}_{2}\right)$, $0.86\left(3 \mathrm{H}, \mathrm{t}, J=6 \mathrm{~Hz}, 17^{27}-\mathrm{CH}_{3}\right)$. MS (FAB) found: $m / z 936$. Calculated for $\mathrm{C}_{56} \mathrm{H}_{80} \mathrm{~N}_{4} \mathrm{O}_{4}^{64} \mathrm{Zn}: \mathrm{M}^{+}, 936$.

\subsubsection{Synthesis of 10,12-pentacosadiyn-1-yl 3-acetyl-3- devinyl-pyropheophorbide-a (1c)}

Esterification of 9 with 10,12-pentacosadiyn-1-ol gave $\mathbf{1 c}$ (93\% yield) after FCC $\left(3 \% \mathrm{Et}_{2} \mathrm{O}-\mathrm{CH}_{2} \mathrm{Cl}_{2}\right)$ and recrystallization $\left(\mathrm{CH}_{2} \mathrm{Cl}_{2}-\mathrm{MeOH}\right) ;{ }^{1} \mathrm{H}-\mathrm{NMR}\left(\mathrm{CDCl}_{3}\right) \delta=10.08$, 9.61, 8.78 (each 1H, s, 5-, 10-, 20-H), 5.33, 5.18 (each $1 \mathrm{H}$, $\left.\mathrm{d}, J=20 \mathrm{~Hz}, 13^{1}-\mathrm{CH}_{2}\right), 4.57(1 \mathrm{H}, \mathrm{br}-\mathrm{q}, J=7 \mathrm{~Hz}, 18-$ $\mathrm{H}), 4.38(1 \mathrm{H}, \mathrm{br}-\mathrm{d}, J=8 \mathrm{~Hz}, 17-\mathrm{H}), 3.90-4.01(2 \mathrm{H}, \mathrm{m}$, $\left.\mathrm{COOCH}_{2}\right), 3.73\left(2 \mathrm{H}, \mathrm{q}, J=7 \mathrm{~Hz}, 8-\mathrm{CH}_{2}\right), 3.72,3.66,3.30$ $\left(3 \mathrm{H}+3 \mathrm{H}+6 \mathrm{H}, \mathrm{s}, 2-, 3^{1}-, 7-, 12-\mathrm{CH}_{3}\right), 2.65-2.78,2.50-2.62$, 2.40-2.23 $\left(1 \mathrm{H}+1 \mathrm{H}+2 \mathrm{H}, \mathrm{m}, 17-\mathrm{CH}_{2} \mathrm{CH}_{2}\right), 2.21,2.18$ (each $\left.2 \mathrm{H}, \mathrm{t}, J=7 \mathrm{~Hz}, \mathrm{CH}_{2} \mathrm{C} \equiv \mathrm{C}-\mathrm{C} \equiv \mathrm{CCH}_{2}\right), 1.84(3 \mathrm{H}, \mathrm{d}, J=7 \mathrm{~Hz}$, $\left.18-\mathrm{CH}_{3}\right), 1.72\left(3 \mathrm{H}, \mathrm{t}, J=7 \mathrm{~Hz}, 8^{1}-\mathrm{CH}_{3}\right), 1.39-1.52(6 \mathrm{H}, \mathrm{m}$, $\left.\mathrm{COOCCH}_{2}, \mathrm{CH}_{2} \mathrm{CC} \equiv \mathrm{C}-\mathrm{C} \equiv \mathrm{CCCH}_{2}\right), 1.17-1.31(28 \mathrm{H}, \mathrm{m}$, $\left.17^{6-10,19-27}-\mathrm{CH}_{2}\right), 0.87\left(3 \mathrm{H}, \mathrm{t}, J=6 \mathrm{~Hz}, 17^{28}-\mathrm{CH}_{3}\right),-0.01$, -2.01 (each $1 \mathrm{H}, \mathrm{s}, \mathrm{NH}$ ).

\subsubsection{Synthesis of 10,12-pentacosadiyn-1-yl bacteriopheophorbide-d (2c)}

Reduction of 1c with $\mathrm{NaBH}_{4}$ gave 2c (56\% yield); ${ }^{1} \mathrm{H}$ $\operatorname{NMR}\left(\mathrm{CDCl}_{3}\right) \delta\left(3^{1} \mathrm{R} / \mathrm{S}=1 / 1\right)=9.64 / 66,9.44,8.50 / 51$ (each 1H, s, 5-, 10-, 20-H), 6.39 (1H, m, 3-CH), 5.20/22, $5.07 / 08$, (each $\left.1 \mathrm{H}, \mathrm{d}, J=20 \mathrm{~Hz}, 13^{1}-\mathrm{CH}_{2}\right), 4.46(1 \mathrm{H}$, br-q, $J=7 \mathrm{~Hz}, 18-\mathrm{H}), 4.25(1 \mathrm{H}, \mathrm{m}, 17-\mathrm{H}), 3.93-4.01$ $\left(2 \mathrm{H}, \mathrm{m}, \mathrm{COOCH}_{2}\right), 3.68\left(2 \mathrm{H}, \mathrm{q}, J=7 \mathrm{~Hz}, 8-\mathrm{CH}_{2}\right), 3.63$, 3.39/40, 3.24 (each $3 \mathrm{H}, \mathrm{s}, 2-$, 7-, 12- $\mathrm{CH}_{3}$ ), 2.57-2.75, 2.45$2.57,2.17-2.35\left(2 \mathrm{H}+1 \mathrm{H}+2 \mathrm{H}, \mathrm{m}, 3^{1}-\mathrm{OH}, 17-\mathrm{CH}_{2} \mathrm{CH}_{2}\right)$, 2.14-2.31 (4H, m, $\left.\mathrm{CH}_{2} \mathrm{C} \equiv \mathrm{C}-\mathrm{C} \equiv \mathrm{CCH}_{2}\right), 2.13(3 \mathrm{H}, \mathrm{d}, J=$ $\left.7 \mathrm{~Hz}, 3^{1}-\mathrm{CH}_{3}\right), 1.77 / 79\left(3 \mathrm{H}, \mathrm{d}, \mathrm{J}=7 \mathrm{~Hz}, 18-\mathrm{CH}_{3}\right), 1.66$ $\left(3 \mathrm{H}, \mathrm{t}, J=7 \mathrm{~Hz}, 8^{1}-\mathrm{CH}_{3}\right), 1.41-1.50\left(6 \mathrm{H}, \mathrm{m}, \mathrm{COOCCH}_{2}\right.$, $\left.\mathrm{CH}_{2} \mathrm{CC} \equiv \mathrm{C}-\mathrm{C} \equiv \mathrm{CCCH}_{2}\right), 1.18-1.38\left(28 \mathrm{H}, \mathrm{m}, 17^{6-10,19-27}\right.$ $\left.\mathrm{CH}_{2}\right), 0.87\left(3 \mathrm{H}, \mathrm{t}, J=6 \mathrm{~Hz}, 17^{28}-\mathrm{CH}_{3}\right), 0.28,-1.85 / 84($ each $1 \mathrm{H}, \mathrm{s}, \mathrm{NH})$.

\subsubsection{Synthesis of zinc 10,12-pentacosadiyn-1-yl bacteriopheophorbide-d (3c)}

Zinc metallation of 2 c gave $3 c$ (80\% yield); VIS (THF) $\lambda_{\max }=645$ (rel., 0.59), 606 (0.11), 564 (0.06), 520 (0.04), $424 \mathrm{~nm}(1.00) ;{ }^{1} \mathrm{H}-\mathrm{NMR}\left(\mathrm{CDCl}_{3}-1 \% \mathrm{C}_{5} \mathrm{D}_{5} \mathrm{~N}\right) \delta\left(3^{1} \mathrm{R} / \mathrm{S}=\right.$ $1 / 1)=9.53 / 56,9.49 / 53,8.28($ each $1 \mathrm{H}, \mathrm{s}, 5-, 10-, 20-\mathrm{H})$ 6.41-6.32 (1H, m, 3-CH), 5.15, 5.03 (each $1 \mathrm{H}, \mathrm{d}, J=20 \mathrm{~Hz}$, $\left.13^{1}-\mathrm{CH}_{2}\right), 4.37(1 \mathrm{H}$, br-q, $J=7 \mathrm{~Hz}, 18-\mathrm{H}), 4.17$ (1H, br-d, $J=7 \mathrm{~Hz}, 17-\mathrm{H}), 3.87-3.96\left(2 \mathrm{H}, \mathrm{m}, \mathrm{COOCH}_{2}\right), 3.73(2 \mathrm{H}$, q, $\left.J=7 \mathrm{~Hz}, 8-\mathrm{CH}_{2}\right), 3.66,3.31 / 33,3.23$ (each $3 \mathrm{H}, \mathrm{s}, 2-, 7-$, $\left.12-\mathrm{CH}_{3}\right), 2.45-2.60,1.86-2.00$ (each $2 \mathrm{H}, \mathrm{m}, 17-\mathrm{CH}_{2} \mathrm{CH}_{2}$ ), 2.13-2.24 (4H, m, $\left.\mathrm{CH}_{2} \mathrm{C} \equiv \mathrm{C}-\mathrm{C} \equiv \mathrm{CCH}_{2}\right), 2.12(3 \mathrm{H}, \mathrm{d}, J=$ $\left.6 \mathrm{~Hz}, 3^{1}-\mathrm{CH}_{3}\right), 1.65-1.80\left(6 \mathrm{H}, \mathrm{m}, 8^{1}-\mathrm{CH}_{3}, 18-\mathrm{CH}_{3}\right), 1.38-$ $1.50\left(6 \mathrm{H}, \mathrm{m}, \mathrm{COOCCH}_{2}, \mathrm{CH}_{2} \mathrm{CC} \equiv \mathrm{C}-\mathrm{C} \equiv \mathrm{CCCH}_{2}\right), 1.20$ $1.33\left(28 \mathrm{H}, \mathrm{m}, 17^{6-10,19-27}-\mathrm{CH}_{2}\right), 0.85(3 \mathrm{H}, \mathrm{t}, J=6 \mathrm{~Hz}$,
$\left.17^{28}-\mathrm{CH}_{3}\right)$. MS (FAB) found: $m / z$ 956. Calculated for $\mathrm{C}_{58} \mathrm{H}_{76} \mathrm{~N}_{4} \mathrm{O}_{4}^{64} \mathrm{Zn}: \mathrm{M}^{+}, 956$.

\subsubsection{Synthesis of 4-(vinylcarbonyloxy)butyl pyropheophorbide-d (1d)}

Esterification of $\mathbf{6}$ with 4-hydroxybutyl acrylate gave 1d (69\% yield) after FCC $\left(1 \% \mathrm{Et}_{2} \mathrm{O}-\mathrm{CH}_{2} \mathrm{Cl}_{2}\right)$ and recrystallization $\left(\mathrm{CH}_{2} \mathrm{Cl}_{2}\right.$-hexane); VIS $\left(\mathrm{CH}_{2} \mathrm{Cl}_{2}\right) \lambda_{\max }=694$ (rel., $0.81), 633$ (0.09), 554 (0.16), 521 (0.15), 428 (1.00), $388 \mathrm{~nm}$ (0.84); ${ }^{1} \mathrm{H}-\mathrm{NMR}\left(\mathrm{CDCl}_{3}\right) \delta=11.56(1 \mathrm{H}, \mathrm{s}, 3-\mathrm{CHO}), 10.32$, 9.63, 8.85 (each 1H, s, 5-, 10-, 20-H), 6.32 (1H, dd, $J=1$, $17 \mathrm{~Hz}, \mathrm{COC}=\mathrm{CH}$ trans to $\mathrm{C}-\mathrm{H}), 6.03(1 \mathrm{H}, \mathrm{dd}, J=10,17 \mathrm{~Hz}$, $\mathrm{COCH}=\mathrm{C}), 5.75(1 \mathrm{H}, \mathrm{dd}, J=1,10 \mathrm{~Hz}, \mathrm{COC}=\mathrm{CH}$ cis to $\mathrm{C}-\mathrm{H}), 5.42,5.13\left(\right.$ each $\left.1 \mathrm{H}, \mathrm{d}, J=20 \mathrm{~Hz}, 13^{1}-\mathrm{CH}_{2}\right), 4.58$ $(1 \mathrm{H}$, br-q, $J=7 \mathrm{~Hz}, 18-\mathrm{H}), 4.40(1 \mathrm{H}, \mathrm{br}-\mathrm{d}, J=8 \mathrm{~Hz}, 17-$ $\mathrm{H})$, 3.93-4.06 (4H, m, $\left.\mathrm{COOCH}_{2} \mathrm{C}_{2} \mathrm{CH}_{2}\right), 3.70-3.78(2 \mathrm{H}, \mathrm{m}$, 8- $\mathrm{CH}_{2}$ ), 3.78, 3.73, 3.33 (each $3 \mathrm{H}, \mathrm{s}, 2-, 7-$, 12- $\mathrm{CH}_{3}$ ), 2.65$2.74,2.42-2.58,2.17-2.36\left(1 \mathrm{H}+1 \mathrm{H}+2 \mathrm{H}, \mathrm{m}, 17-\mathrm{CH}_{2} \mathrm{CH}_{2}\right)$, $1.85\left(3 \mathrm{H}, \mathrm{d}, J=7 \mathrm{~Hz}, 18-\mathrm{CH}_{3}\right), 1.72(3 \mathrm{H}, \mathrm{t}, J=7 \mathrm{~Hz}$, $\left.8^{1}-\mathrm{CH}_{3}\right) 1.54-1.65\left(4 \mathrm{H}, \mathrm{m}, \mathrm{COOCCH}_{2} \mathrm{CH}_{2}\right), 0.24,-1.83$ (each $1 \mathrm{H}, \mathrm{s}, \mathrm{NH})$. MS (FAB) found; $m / z 662$. Calculated for $\mathrm{C}_{39} \mathrm{H}_{42} \mathrm{~N}_{4} \mathrm{O}_{6}: \mathrm{M}^{+}, 662$.

\subsubsection{Synthesis of 4-(vinylcarbonyloxy)butyl 3- hydroxymethyl-3-devinyl-pyropheophorbide-a (2d)}

Reduction of $\mathbf{1 d}$ with $\mathrm{t}-\mathrm{BuNH}_{2} \mathrm{BH}_{3}$ gave $2 \mathbf{d}$ ( $86 \%$ yield); VIS $\left(\mathrm{CH}_{2} \mathrm{Cl}_{2}\right) \lambda_{\max }=662$ (rel., 0.48), 605 (0.08), 535 (0.09), 505 (0.10), $410 \mathrm{~nm}(1.00) ;{ }^{1} \mathrm{H}-\mathrm{NMR}\left(\mathrm{CDCl}_{3}\right) \delta=9.45,9.43,8.56$ (each $1 \mathrm{H}, \mathrm{s}, 5-, 10-, 20-\mathrm{H}), 6.31(1 \mathrm{H}, \mathrm{dd}, J=2,17 \mathrm{~Hz}$, $\mathrm{COC}=\mathrm{CH}$ trans to $\mathrm{C}-\mathrm{H}), 6.01(1 \mathrm{H}, \mathrm{dd}, J=10,17 \mathrm{~Hz}$, $\mathrm{COCH}=\mathrm{C}), 5.90,5.87\left(\right.$ each $\left.1 \mathrm{H}, \mathrm{d}, J=13 \mathrm{~Hz}, 3-\mathrm{CH}_{2}\right) 5.74$ $(1 \mathrm{H}, \mathrm{dd}, J=2,10 \mathrm{~Hz}, \mathrm{COC}=\mathrm{CH}$ cis to $\mathrm{C}-\mathrm{H}), 5.22,5.08$ (each $\left.1 \mathrm{H}, \mathrm{d}, J=19 \mathrm{~Hz}, 13^{1}-\mathrm{CH}_{2}\right), 4.47(1 \mathrm{H}, \mathrm{dq}, J=2,8 \mathrm{~Hz}$, $18-\mathrm{H}), 4.28$ (1H, dt, $J=8,2 \mathrm{~Hz}, 17-\mathrm{H}), 3.90-3.95,3.78-3.79$ $\left(3 \mathrm{H}+1 \mathrm{H}, \mathrm{m}, \mathrm{COOCH}_{2} \mathrm{C}_{2} \mathrm{CH}_{2}\right), 3.68(2 \mathrm{H}, \mathrm{q}, J=8 \mathrm{~Hz}, 8-$ $\mathrm{CH}_{2}$ ), 3.63, 3.41, 3.25 (each $\left.3 \mathrm{H}, \mathrm{s}, 2-, 7-, 12-\mathrm{CH}_{3}\right), 2.62-2.67$, $2.44-2.49,2.30-2.36,2.17-2.24\left(1 \mathrm{H}+1 \mathrm{H}+2 \mathrm{H}+1 \mathrm{H}, \mathrm{m}, 3^{1}-\right.$ $\left.\mathrm{OH}, 17-\mathrm{CH}_{2} \mathrm{CH}_{2}\right), 1.80\left(3 \mathrm{H}, \mathrm{d}, J=8 \mathrm{~Hz}, 18-\mathrm{CH}_{3}\right), 1.68(3 \mathrm{H}$, $\left.\mathrm{t}, J=8 \mathrm{~Hz}, 8^{1}-\mathrm{CH}_{3}\right), 1.39-1.41\left(4 \mathrm{H}, \mathrm{m}, \mathrm{COOCCH}_{2} \mathrm{CH}_{2}\right)$, $0.24,-1.83$ (each $1 \mathrm{H}, \mathrm{s}, \mathrm{NH}$ ). MS (FAB) found; $m / z 664$. Calculated for $\mathrm{C}_{39} \mathrm{H}_{44} \mathrm{~N}_{4} \mathrm{O}_{6}: \mathrm{M}^{+}, 664$.

\subsubsection{Synthesis of zinc 4-(vinylcarbonyloxy)butyl 3- hydroxymethyl-3-devinyl-pyropheophorbide-a (3d)}

Zinc metallation of $\mathbf{2 d}$ gave $\mathbf{3 d}$ (83\% yield); VIS (THF) $\lambda_{\max }=646$ (rel., 0.75), 600 (0.10), 566 (0.05), 521 (0.03), 424 (1.00), $404 \mathrm{~nm}(0.56) ;{ }^{1} \mathrm{H}-\mathrm{NMR}\left(\mathrm{CDCl}_{3}-1 \% \mathrm{C}_{5} \mathrm{D}_{5} \mathrm{~N}\right)$ $\delta=9.55,9.32,8.33($ each $1 \mathrm{H}, \mathrm{s}, 5-, 10-, 20-\mathrm{H}), 6.31(1 \mathrm{H}$, $\mathrm{dd}, J=2,17 \mathrm{~Hz}, \mathrm{COC}=\mathrm{CH}$ trans to $\mathrm{C}-\mathrm{H}), 6.02(1 \mathrm{H}, \mathrm{dd}$, $J=10,17 \mathrm{~Hz}, \mathrm{COCH}=\mathrm{C}), 5.82\left(2 \mathrm{H}, \mathrm{s}, 3-\mathrm{CH}_{2}\right) 5.76(1 \mathrm{H}$, $\mathrm{dd}, J=2,10 \mathrm{~Hz}, \mathrm{COC}=\mathrm{CH}$ cis to $\mathrm{C}-\mathrm{H}), 5.16,5.05$ (each $\left.1 \mathrm{H}, \mathrm{d}, J=19 \mathrm{~Hz}, 13^{1}-\mathrm{CH}_{2}\right), 4.39(1 \mathrm{H}, \mathrm{dd}, J=2,7 \mathrm{~Hz}, 18-$ $\mathrm{H}), 4.19(1 \mathrm{H}, \mathrm{dt}, J=7,2 \mathrm{~Hz}, 17-\mathrm{H}), 4.00-4.02,3.93-3.96$, 3.85-3.89 $\left(2 \mathrm{H}+1 \mathrm{H}+1 \mathrm{H}, \mathrm{m}, \mathrm{COOCH}_{2} \mathrm{C}_{2} \mathrm{CH}_{2}\right), 3.73(2 \mathrm{H}$, q, $\left.J=8 \mathrm{~Hz}, 8-\mathrm{CH}_{2}\right), 3.67,3.32,3.22($ each $3 \mathrm{H}, \mathrm{s}, 2-, 7-$, $\left.12-\mathrm{CH}_{3}\right), 2.51-2.55,2.27-2.35,1.92-1.96,(1 \mathrm{H}+2 \mathrm{H}+1 \mathrm{H}$, 


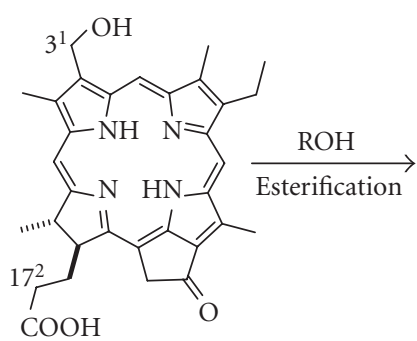

10



Monomer 2


FIgURE 2: Esterification of $3^{1}$-hydroxy- $17^{2}$-carboxy-chlorin 10 with alcohol (ROH).

$\left.\mathrm{m}, 17-\mathrm{CH}_{2} \mathrm{CH}_{2}\right), 1.70\left(3 \mathrm{H}, \mathrm{d}, J=8 \mathrm{~Hz}, 18-\mathrm{CH}_{3}\right), 1.68(3 \mathrm{H}, \mathrm{t}$, $\left.J=8 \mathrm{~Hz}, 8^{1}-\mathrm{CH}_{3}\right), 1.47-1.53\left(4 \mathrm{H}, \mathrm{m}, \mathrm{COOCCH}_{2} \mathrm{CH}_{2}\right) . \mathrm{MS}$ (FAB) found; $m / z$ 726. Calculated for $\mathrm{C}_{39} \mathrm{H}_{42} \mathrm{~N}_{4} \mathrm{O}_{6}^{64} \mathrm{Zn}: \mathrm{M}^{+}$, 726.

\section{RESULTS AND DISCUSSION}

\subsection{Synthesis of zinc bacteriochlorophyll-d derivatives possessing a diacetylene moiety}

Esterification of $3^{1}$-hydroxy-172-carboxy-chlorin 10 with alcohol $(\mathrm{ROH})$ gave oligomeric chlorins including dimer as byproducts besides the desired $17^{2}$-COOR ester 2 (see Figure 2). To prevent such undesired condensation affording $17^{2}$-COOC $\left(3^{1}\right)$ esters, the reactive $3^{1}$-hydroxy group was protected by its oxidation before esterification. Starting carboxylic acids 6 and 9 possessing the 3-carbonyl group were prepared by modifying methyl pyropheophorbide- $a$ (4) obtained from chlorophyll-a (see Scheme 1). 3-Formyl-17 ${ }^{2}$ carboxy-chlorin 6 was synthesized according to reported procedures [18] as in $\mathbf{4} \rightarrow \mathbf{5} \rightarrow \mathbf{6}$ : the 3 -vinyl group of $\mathbf{4}$ was oxidatively cleaved to the corresponding 3 -formyl group and successively the methyl ester of $\mathbf{5}$ was hydrolyzed to yield 6. 3Acetyl-172-methoxycarbonyl-chlorin 8 was afforded from 4 through hydration of the 3 -vinyl group in 4 and oxidation of the resulting 1-hydroxyethyl group in 7 to the 3 -acetyl group in $8[19,20]$ and hydrolyzed by action of an acid [21] to give the corresponding carboxylic acid 9 (94\% yield).

As a polymerizable functional group, a diacetylene moiety was first introduced in the $17^{2}$-ester. Carboxylic acids 6 and 9 reacted with 10,12-pentacosadiyn-1-ol by action of water-soluble carbodiimide (EDC) as a dehydrating condensation reagent in the presence of DMAP as a condensation promoter $[9,22]$ to afford esters $1 \mathrm{a}$ and $1 \mathrm{c}$ in good yields (see Scheme 2). The 3-formyl group in 1a was selectively reduced by tert-butylamine borane complex [23] to give 2 a possessing the 3 -hydroxymethyl group. The 3 -acetyl group in 1c was reduced by more reactive sodium borohydride, because a more sterically hindered keto group is less reactive than a formyl group. $\mathrm{NaBH}_{4}$ can reduce the $13-\mathrm{C}=\mathrm{O}$ in a molecule and the reduction must be stopped just after visible absorption, and TLC analyses indicate a disappearance of the 3-acetyl group. It is noteworthy that the reductivity of peripheral carbonyl groups on the chlorin $\pi$-moiety decreases in the order, 3-CHO > 3- $\mathrm{COCH}_{3}>13-\mathrm{CO}$.

Zinc complexes $3 a$ and $3 c$ were obtained from free bases $\mathbf{2 a}$ and $\mathbf{2 c}$ by standard procedures [9]. 3-(1-Hydroxyethyl)chlorin $3 \mathrm{c}$ was a $3^{1}$-epimeric mixture and the $1: 1$ mixture was used for the following experiments. As a nonpolymerizable reference compound, zinc 3-hydroxymethyl-chlorin $\mathbf{3 b}$ having tetracosanyl ester was prepared similarly to the synthesis of 3a. All the synthetic chlorins were characterized by their ${ }^{1} \mathrm{H}-\mathrm{NMR}$, visible, IR, and/or mass spectra.

\subsection{Self-aggregation of zinc bacteriochlorophyll-d derivatives possessing a diacetylene moiety}

\subsubsection{In a nonpolar organic solvent}

In THF, 3a gave sharp $\mathrm{Q}_{\mathrm{y}}$ and Soret peaks at 646 and $424 \mathrm{~nm}$ (dashed line of Figure 3(a)). The species was monomeric and five-coordinated zinc complex with a THF molecule as an axial ligand, compared with previous data $[7,9,21,23]$. When the solution was diluted with hexane, broadened and redshifted absorption bands were observed. In $0.1 \%$ (v/v) THFhexane, 739- and 450-nm bands were mainly observed and small monomeric peaks remained (solid line of Figure 3(a)), indicating that most of 3 a self-aggregated to form a similar oligomer to chlorosomal J-aggregates [7, 9, 21, 23]. In the nonpolar organic solvent, $3 \mathbf{b}$ lacking a polymerizable group gave the same oligomeric absorption peaks at 739 and $450 \mathrm{~nm}$. These results clearly indicate that such long hydrocarbon chains as well as the rigid diacetylene moiety in the 17-propionate did not disturb self-aggregation of zinc $3^{1}$-hydroxy- $13^{1}$-oxo-chlorins. 


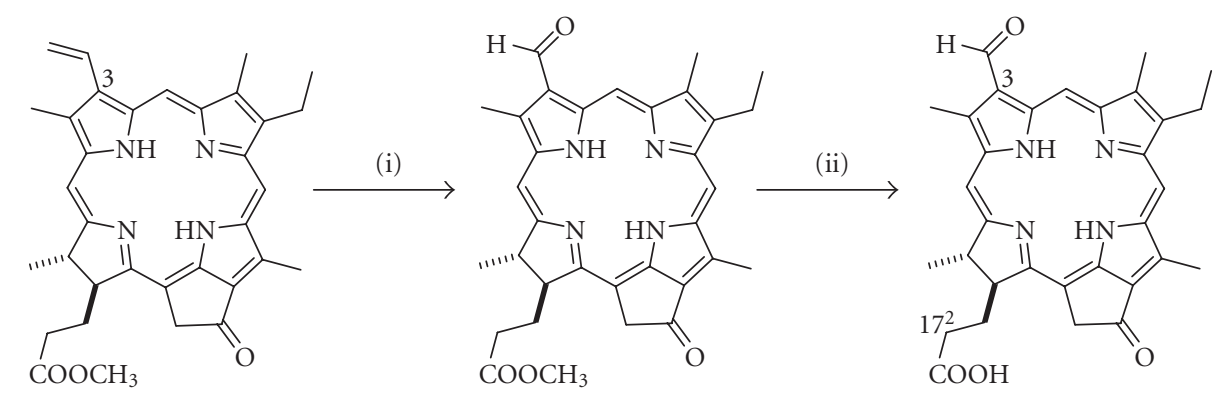

Methyl pyropheophorbide- $a$ (4)

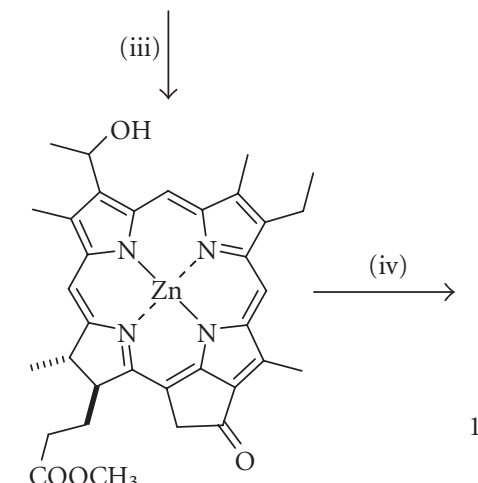

$\mathrm{COOCH}_{3}$

Methyl bacterio-

pheophorbide- $d$ (7)
Methyl pyropheophorbide- $d(5)$
Pyropheophorbide- $d$ (6)

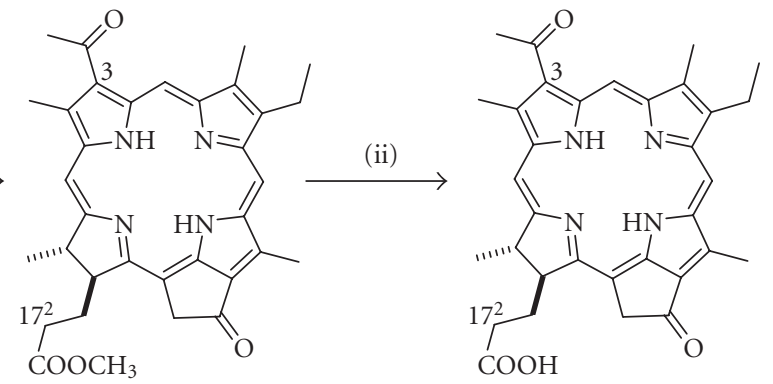

Methyl 3-acetyl-pyropheophorbide- $a(8)$
3-Acetyl-pyropheophorbide- $a$ (9)

Scheme 1: Synthesis of pyropheophoribides: (i) $\mathrm{OsO}_{4}-\mathrm{NaIO}_{4}$ /aqueous $\mathrm{AcOH}-\mathrm{THF}$; (ii) conc. $\mathrm{HCl}$; (iii) $\mathrm{HBr} / \mathrm{AcOH} \mathrm{H}_{2} \mathrm{O}, \mathrm{CH}_{2} \mathrm{~N}_{2} / \mathrm{Et}_{2} \mathrm{O}$; (iv) $\mathrm{Pr}_{4} \mathrm{NRuO}_{4}-\mathrm{Me}(\mathrm{O}) \mathrm{N}\left(\mathrm{CH}_{2} \mathrm{CH}_{2}\right)_{2} \mathrm{O} / \mathrm{CH}_{2} \mathrm{Cl}_{2}$.

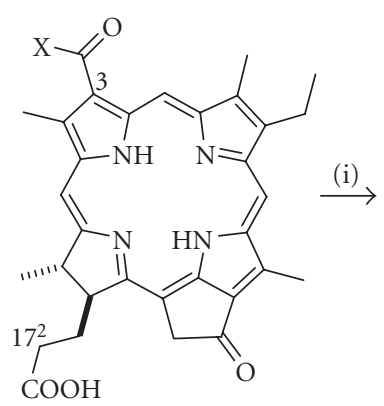

6: $\mathrm{X}=\mathrm{H}$

9: $\mathrm{X}=\mathrm{CH}_{3}$

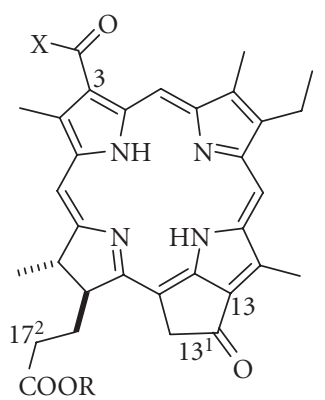

1a: $\mathrm{X}=\mathrm{H}, \mathrm{R}=\left(\mathrm{CH}_{2}\right)_{9}-$

$\mathrm{C} \equiv \mathrm{C}-\mathrm{C} \equiv \mathrm{C}\left(\mathrm{CH}_{2}\right)_{12} \mathrm{H}$

1b: $\mathrm{X}=\mathrm{H}, \mathrm{R}=\left(\mathrm{CH}_{2}\right)_{24} \mathrm{H}$

1c: $\mathrm{X}=\mathrm{CH}_{3}, \mathrm{R}=\left(\mathrm{CH}_{2}\right)_{9}-$

$\mathrm{C} \equiv \mathrm{C}-\mathrm{C} \equiv \mathrm{C}\left(\mathrm{CH}_{2}\right)_{12} \mathrm{H}$

1d: $\mathrm{X}=\mathrm{H}, \mathrm{R}=\left(\mathrm{CH}_{2}\right)_{4}-$

$\mathrm{OCOCH}=\mathrm{CH}_{2}$



2a: $\mathrm{X}=\mathrm{H}, \mathrm{R}=\left(\mathrm{CH}_{2}\right)_{9}-$

$\mathrm{C} \equiv \mathrm{C}-\mathrm{C} \equiv \mathrm{C}\left(\mathrm{CH}_{2}\right)_{12} \mathrm{H}$

2b: $\mathrm{X}=\mathrm{H}, \mathrm{R}=\left(\mathrm{CH}_{2}\right)_{24} \mathrm{H}$

2c: $\mathrm{X}=\mathrm{CH}_{3}, \mathrm{R}=\left(\mathrm{CH}_{2}\right)_{9}-$

$\mathrm{C} \equiv \mathrm{C}-\mathrm{C} \equiv \mathrm{C}\left(\mathrm{CH}_{2}\right)_{12} \mathrm{H}$

2d: $\mathrm{X}=\mathrm{H}, \mathrm{R}=\left(\mathrm{CH}_{2}\right)_{4}-$

$\mathrm{OCOCH}=\mathrm{CH}_{2}$

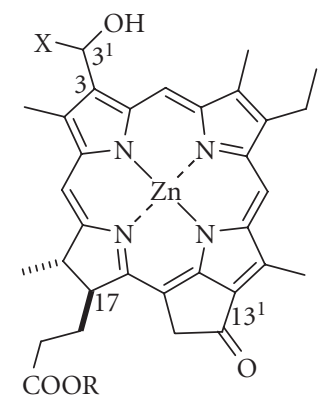

3a: $\mathrm{X}=\mathrm{H}, \mathrm{R}=\left(\mathrm{CH}_{2}\right)_{9}-$

$\mathrm{C} \equiv \mathrm{C}-\mathrm{C} \equiv \mathrm{C}\left(\mathrm{CH}_{2}\right)_{12} \mathrm{H}$

3b: $\mathrm{X}=\mathrm{H}, \mathrm{R}=\left(\mathrm{CH}_{2}\right)_{24} \mathrm{H}$

3c: $\mathrm{X}=\mathrm{CH}_{3}, \mathrm{R}=\left(\mathrm{CH}_{2}\right)_{9}-$

$\mathrm{C} \equiv \mathrm{C}-\mathrm{C} \equiv \mathrm{C}\left(\mathrm{CH}_{2}\right)_{12} \mathrm{H}$

3d: $\mathrm{X}=\mathrm{H}, \mathrm{R}=\left(\mathrm{CH}_{2}\right)_{4}$

$\mathrm{OCOCH}=\mathrm{CH}_{2}$

Scheme 2: Synthesis of zinc bacteriochlorophyll- $d$ derivatives: (i) $\mathrm{ROH}, \mathrm{EDC}-\mathrm{DMAP} / \mathrm{CH}_{2} \mathrm{Cl}_{2} ;$ (ii) $\mathrm{t}-\mathrm{BuNH}_{2} \mathrm{BH}_{3} / \mathrm{CH}_{2} \mathrm{Cl}_{2}$ for $\mathrm{X}=\mathrm{H}$, $\mathrm{NaBH}_{4} / \mathrm{MeOH}-\mathrm{CH}_{2} \mathrm{Cl}_{2}$ for $\mathrm{X}=\mathrm{CH}_{3}$; (iii) $\mathrm{Zn}(\mathrm{OAc})_{2} \cdot 2 \mathrm{H}_{2} \mathrm{O} / \mathrm{MeOH}-\mathrm{CH}_{2} \mathrm{Cl}_{2}$.

Secondary alcohol $3 \mathbf{c}$, one of zinc $3^{1}$-hydroxy-13 $3^{1}$-oxochlorins, self-aggregated in $0.1 \%(\mathrm{v} / \mathrm{v})$ THF-hexane to give electronic absorption bands characteristic of its J-aggregates (see Figure 3(b)). The red-shift values in peak positions by self-aggregation of $3 \mathrm{c}$ were smaller than those in 3a: typically, Qy-peak shifts were 1930 and $1260 \mathrm{~cm}^{-1}$ for 3a and $3 c$, respectively. This difference is ascribable to the steric effect around the $3^{1}-\mathrm{OH}$ which connected the molecules in the self-aggregates, that is, the less steric $\mathrm{OH}$ in primary alcohol 3a can coordinate to the zinc metal and hydrogen-bond with the 13-carbonyl group more tightly to give larger $\pi-\pi$ interaction of chlorin chromophores in the supramolecule than the secondary alcoholic $\mathrm{OH}$ in $3 \mathrm{c}$ [24].

\subsubsection{In the solid state}

The above nonpolar organic solutions containing selfaggregates of $3 \mathbf{a}-\mathbf{c}$ were cast on a quartz glass plate and all 


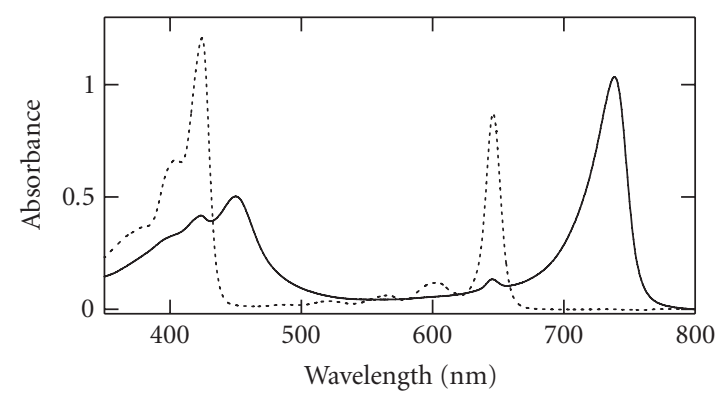

(a)

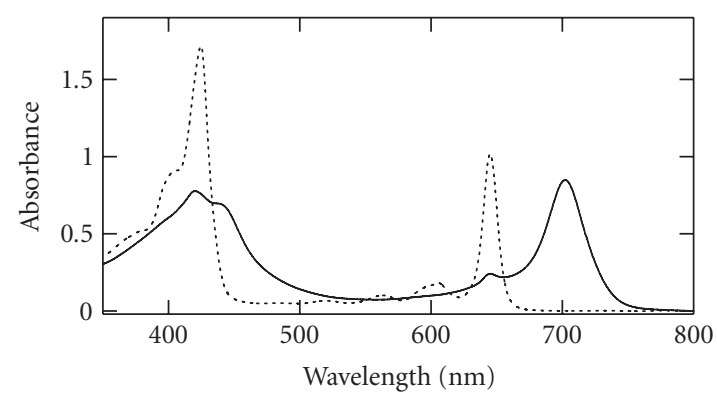

(b)

FIgURe 3: Visible absorption spectra of $3 a$ (a) and $3 \mathrm{c}$ (b) in THF (dashed line) and $1 \%(\mathrm{v} / \mathrm{v})$ THF-hexane (solid line) at $25^{\circ} \mathrm{C}$ (ca. $15 \mu \mathrm{M})$.

the solvents were evaporated to afford the solid films. The absorption spectra of the resulting films possessing ca. 735and 450-nm peaks were nearly the same as those in a solution (data not shown). The solid states of $\mathbf{3 a}-\mathbf{c}$ still gave their J-aggregates similarly to those in a nonpolar organic solvent.

Preliminarily, the self-aggregated films were irradiated with an ultraviolet light $(254 \mathrm{~nm})$ or heated at $180^{\circ} \mathrm{C}$ for polymerization of the diacetylene groups in $3 \mathrm{a} / \mathrm{c}$, so that no polymers were given. The resulting films could not afford an appropriate orientation of diacetylene moieties in the solid state for their topochemical polymerization [25]. Further investigation is underway to produce poly(diacetylene) in the esters of self-aggregates.

\subsection{Synthesis of zinc bacteriochlorophyll-d derivative possessing an acrylate moiety and its self-aggregation}

An acrylate substituent was utilized for a polymerizable group in the 17 -propionate. The synthetic route to $\mathbf{3} \mathbf{d}$ possessing an acrylate moiety is the same as that to $3 \mathbf{a}$ (see Scheme 2). During the preparation procedures $(\mathbf{6} \rightarrow \mathbf{1 d} \rightarrow$ $2 \mathbf{d} \rightarrow \mathbf{3 d}$ ), no polymerization of the acrylate occurred.

Synthetic compound $\mathbf{3 d}$ self-aggregated in benzene $\left(\lambda_{\max }=\right.$ ca. $\left.735 / 450 \mathrm{~nm}\right)$ as well as in hexane to give chlorosomal J-aggregates (data not shown). The acrylate moiety in the ester terminal did not prevent such self-aggregation. Preliminarily, radical polymerization of $\mathbf{3 d}$ in benzene was examined using $\alpha, \alpha^{\prime}$-azobisisobutyronitrile as an initiator.
After heating at $80^{\circ} \mathrm{C}$ for 1 hour, oligomers of $\mathbf{3 d}$ were detected from gel permeation chromatography. The supramolecular structures of the resulting oligomers are being elucidated in our laboratory.

\section{ACKNOWLEDGMENTS}

We thank Mr. Toru Takimoto of Ritsumeikan University for his early contribution to this research. This work was partially supported by Grants-in-Aid for Scientific Research (no. 17029065) on Priority Areas (417) from the Ministry of Education, Culture, Sports, Science and Technology (MEXT) of the Japanese Government and by Scientific Research (B) (no. 15350107) from the Japan Society for the Promotion of Science (JSPS), as well as by the "Academic Frontier" Project for Private Universities: matching fund subsidy from MEXT, 2003-2007.

\section{REFERENCES}

[1] R. E. Blankenship, J. M. Olson, and M. Miller, "Antenna complexes from green photosynthetic bacteria," in Anoxygenic Photosynthetic Bacteria, R. E. Blankenship, M. T. Madigan, and C. E. Bauer, Eds., pp. 399-435, Kluwer Academic, Dordrecht, The Netherlands, 1995.

[2] H. Tamiaki, "Supramolecular structure in extramembraneous antennae of green photosynthetic bacteria," Coordination Chemistry Reviews, vol. 148, pp. 183-197, 1996.

[3] J. M. Olson, "Chlorophyll organization and function in green photosynthetic bacteria," Photochemistry and Photobiology, vol. 67, no. 1, pp. 61-75, 1998.

[4] T. S. Balaban, H. Tamiaki, and A. R. Holzwarth, "Chlorins programmed for self-assembly," in Supramolecular Chemistry of Dyes and Pigments, vol. 258 of Topics in Current Chemistry, pp. 1-38, Springer, Heidelberg, Germany, 2005.

[5] H. Tamiaki, "Self-aggregates of natural and modified chlorophylls as photosynthetic light-harvesting antenna systems: substituent effect on the B-ring," Photochemical and Photobiological Sciences, vol. 4, no. 9, pp. 675-680, 2005.

[6] A. R. Holzwarth and K. Schaffner, "On the structure of bacteriochlorophyll molecular aggregates in the chlorosomes of green bacteria. a molecular modelling study," Photosynthesis Research, vol. 41, no. 1, pp. 225-233, 1994.

[7] H. Tamiaki, S. Miyata, Y. Kureishi, and R. Tanikaga, "Aggregation of synthetic zinc chlorins with several esterified alkyl chains as models of bacteriochlorophyll-c homologs," Tetrahedron, vol. 52, no. 38, pp. 12421-12432, 1996.

[8] T. Miyatake and H. Tamiaki, "Self-aggregates of bacteriochlorophylls- $c, d$ and $e$ in a light-harvesting antenna system of green photosynthetic bacteria: effect of stereochemistry at the chiral 3-(1-hydroxyethyl) group on the supramolecular arrangement of chlorophyllous pigments," Journal of Photochemistry and Photobiology C: Photochemistry Reviews, vol. 6, no. 2-3, pp. 89-107, 2005.

[9] R. Shibata and H. Tamiaki, "Self-aggregation of synthetic zinc chlorophyll derivative possessing a perfluoroalkyl group in a fluorinated solvent," Bioorganic and Medicinal Chemistry, vol. 14, no. 7, pp. 2235-2241, 2006.

[10] H. Tamiaki, R. Shibata, and T. Mizoguchi, to appear in Photochemistry and Photobiology. 
[11] V. Huber, M. Katterle, M. Lysetska, and F. Würthner, "Reversible self-organization of semisynthetic zinc chlorins into well-defined rod antennae," Angewandte Chemie International Edition, vol. 44, no. 20, pp. 3147-3151, 2005.

[12] N.-U. Frigaard, A. G. M. Chew, H. Li, J. A. Maresca, and D. A. Bryant, "Chlorobium tepidum: insights into the structure, physiology, and metabolism of a green sulfur bacterium derived from the complete genome sequence," Photosynthesis Research, vol. 78, no. 2, pp. 93-117, 2003.

[13] Y. Saga, T. Miyatake, and H. Tamiaki, "Self-Assembly of synthetic zinc chlorins in a silicate micelle prepared by sol-gel process," Bioorganic and Medicinal Chemistry Letters, vol. 12, no. 8, pp. 1229-1231, 2002.

[14] Y. Saga, S. Akai, T. Miyatake, and H. Tamiaki, "Excitation energy transfer from self-aggregates of zinc chlorins to a bacteriochlorin in a silicate nanocapsule," Chemistry Letters, vol. 33, no. 5, pp. 544-545, 2004.

[15] Y. Saga, S. Akai, T. Miyatake, and H. Tamiaki, "Self-assembly of natural light-harvesting bacteriochlorophylls of green sulfur photosynthetic bacteria in silicate capsules as stable models of chlorosomes," Bioconjugate Chemistry, vol. 17, no. 4, pp. 988-994, 2006.

[16] A. Mueller and D. F. O'Brien, "Supramolecular materials via polymerization of mesophases of hydrated amphiphiles," Chemical Reviews, vol. 102, no. 3, pp. 727-757, 2002.

[17] W. Jin, T. Fukushima, A. Kosaka, M. Niki, N. Ishii, and T. Aida, "Controlled self-assembly triggered by olefin metathesis: cross-linked graphitic nanotubes from an amphiphilic hexaperi-hexabenzocoronene," Journal of the American Chemical Society, vol. 127, no. 23, pp. 8284-8285, 2005.

[18] N. Kosaka and H. Tamiaki, "Synthesis of a novel cyclic chlorophyll hetero-dyad as a model compound for stacked chlorophylls found in photosynthetic systems," European Journal of Organic Chemistry, no. 11, pp. 2325-2330, 2004.

[19] H. Tamiaki, S. Yagai, and T. Miyatake, "Synthetic zinc tetrapyrroles complexing with pyridine as a single axial ligand," Bioorganic and Medicinal Chemistry, vol. 6, no. 11, pp. 2171-2178, 1998.

[20] H. Tamiaki, M. Kouraba, K. Takeda, S.-I. Kondo, and R. Tanikaga, "Asymmetric synthesis of methyl bacteriopheophorbide$d$ and analogues by stereoselective reduction of the 3-acetyl to the 3-(1-hydroxyethyl) group," Tetrahedron Asymmetry, vol. 9, no. 12, pp. 2101-2111, 1998.

[21] T. Miyatake, H. Tamiaki, A. R. Holzwarth, and K. Schaffner, "Artificial light-harvesting antennae: singlet excitation energy transfer from zinc chlorin aggregate to bacteriochlorin in homogeneous hexane solution," Photochemistry and Photobiology, vol. 69, no. 4, pp. 448-456, 1999.

[22] T. Miyatake, H. Tamiaki, H. Shinoda, M. Fujiwara, and T. Matsushita, "Synthesis and self-assembly of amphiphilic zinc chlorins possessing a $3^{1}$-hydroxy group," Tetrahedron, vol. 58, no. 50, pp. 9989-10000, 2002.

[23] H. Tamiaki, M. Amakawa, Y. Shimono, R. Tanikaga, A. R. Holzwarth, and K. Schaffner, "Synthetic zinc and magnesium chlorin aggregates as models for supramolecular antenna complexes in chlorosomes of green photosynthetic bacteria," Photochemistry and Photobiology, vol. 63, no. 1, pp. 92-99, 1996.

[24] S. Yagai, T. Miyatake, Y. Shimono, and H. Tamiaki, "Supramolecular structure of self-assembled synthetic zinc- $13^{1}$-oxochlorins possessing a primary, secondary or tertiary alcoholic $3^{1}$-hydroxyl group: visible spectroscopic and molecular modeling studies," Photochemistry and Photobiology, vol. 73, no. 2, pp. 153-163, 2001.

[25] V. Enkelmann, "Structural aspects of the topochemical polymerization of diacetylenes," in Advances in Polymer Science: Polydiacetylenes, vol. 63, pp. 91-136, Springer, Berlin, Germany, 1984. 


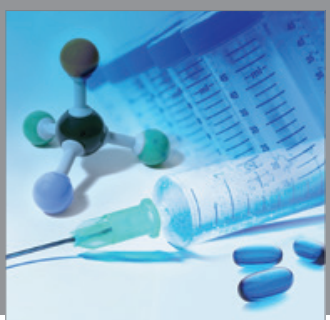

International Journal of

Medicinal Chemistry

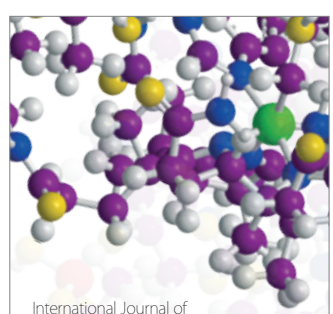

Carbohydrate Chemistry

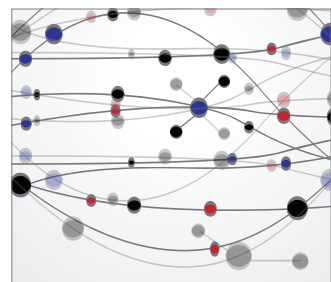

The Scientific World Journal
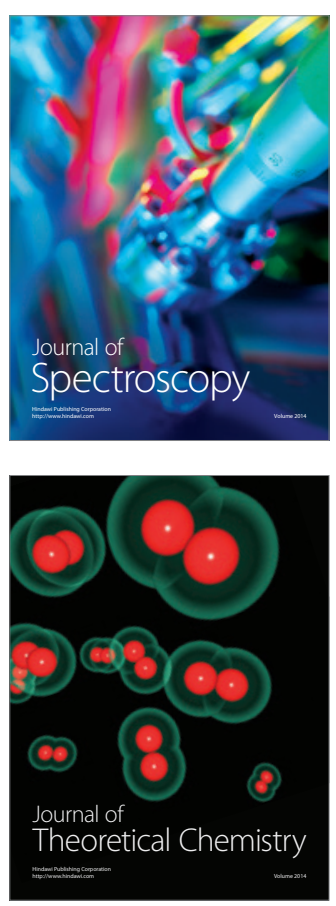
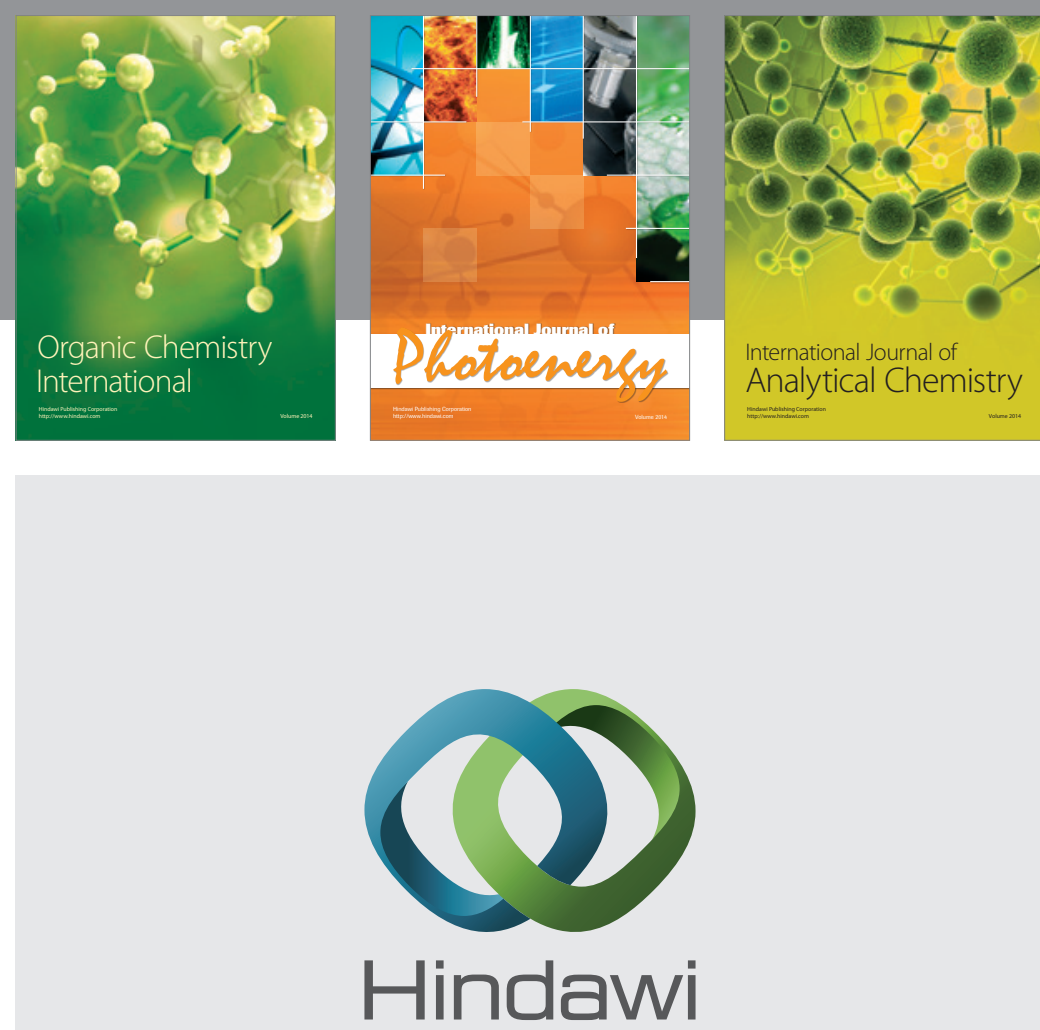

Submit your manuscripts at

http://www.hindawi.com


Journal of

Applied Chemistry

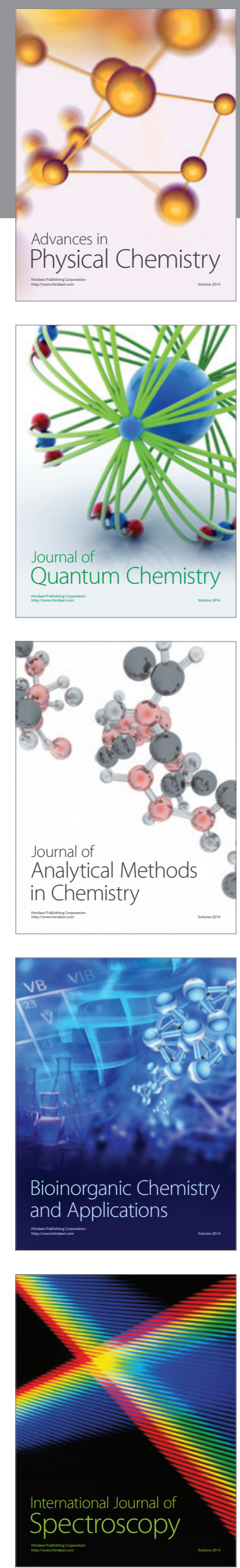